\title{
The Critical Need to Promote Research of Aging and Aging-related Diseases to Improve Health and Longevity of the Elderly Population
}

\author{
Kunlin Jin ${ }^{1,2}$, James W. Simpkins ${ }^{1,3}$, Xunming Ji ${ }^{1,4}$, Miriam Leis ${ }^{1,5}$, Ilia Stambler ${ }^{1,6}$ \\ ${ }^{1}$ Executive Committee, International Society on Aging and Disease (ISOAD), Fort Worth, TX76137, USA \\ ${ }^{2}$ Department of Pharmacology and Neuroscience, University of North Texas Health Science Center, Fort Worth, \\ TX76137, USA. \\ ${ }^{3}$ Center for Basic and Translational Stroke Research, West Virginia University, West Virginia, USA. \\ ${ }^{4}$ Department of Neurosurgery, Xuanwu Hospital, Capital Medical University, Beijing, China. \\ ${ }^{5}$ Fraunhofer Society for the Advancement of Applied Research, Munich, Germany. \\ ${ }^{6}$ Department of Science, Technology and Society, Bar Ilan University, Ramat Gan, Israel.
}

[Received November 14, 2015; Revised December 9, 2014; Accepted December 10, 2014]

\begin{abstract}
Due to the aging of the global population and the derivative increase in aging-related noncommunicable diseases and their economic burden, there is an urgent need to promote research on aging and aging-related diseases as a way to improve healthy and productive longevity for the elderly population. To accomplish this goal, we advocate the following policies: 1) Increasing funding for research and development specifically directed to ameliorate degenerative aging processes and to extend healthy and productive lifespan for the population; 2) Providing a set of incentives for commercial, academic, public and governmental organizations to foster engagement in such research and development; and 3) Establishing and expanding coordination and consultation structures, programs and institutions involved in aging-related research, development and education in academia, industry, public policy agencies and at governmental and supragovernmental levels.
\end{abstract}

Key words: aging, aging-related diseases, health, longevity, elderly, population

The challenge of the aging society and potential solutions

Over the past decades, the average life expectancy has increased globally, reaching a world-wide average of about 70 years in 2014 (6 years longer than in 1990) and around 80 in the developed countries (compared to about 50 years in the developed countries in the early $20^{\text {th }}$ century). This development has been achieved especially due to improvements in sanitation, medical advances, rising living standards and a decline in child mortality.
Currently, while the longest life expectancies are still found in the "developed" countries, the fastest and largest increase has been recorded in the "developing" world. Considering the demographics of the world population, between 2000 and 2050 the proportion of people over 60 years will double from about $11 \%$ to $22 \%$, which, in absolute terms, means an increase from 605 million to 2 billion people [1-3].

Although the increasing life expectancy generally reflects positive human development, new challenges are arising. They stem from the fact that growing older is still

*Correspondence should be addressed to: Executive Committee, International Society on Aging and Disease (ISOAD), Fort Worth, TX76137, USA. E-mail: isoad@,ispad.org 
inherently associated with biological and cognitive degeneration, although the severity and speed of cognitive decline, physical frailty and psychological impairment can vary between individuals.

Nonetheless, degenerative aging processes are the major underlying cause for non-communicable diseases (NCDs), including cancer, ischemic heart disease, stroke, type 2 diabetes, Alzheimer's disease and others. Mental health deterioration due to chronic neurodegenerative diseases represents the largest cause of disability in the world, responsible for over $20 \%$ of years lived with disability. Hence, major efforts must be directed toward their alleviation. According to the World Health Organization's (WHO) "Draft Twelfth General Programme of Work" (April 13, 2013), one of WHO leadership priorities is "Addressing the challenge of noncommunicable diseases" [4], and addressing aging fits into this definition and is even necessary to accomplish this priority goal.

Aging also increases the risk of morbidity and mortality from infectious diseases like pneumonia and influenza. Moreover, the susceptibility to injury and trauma (such as falls and concussions), due to the impairment of balance and mental state, and even falling victim to violence, are strongly increased by the aging process. Also, the processes of aging exacerbate and reinforce the effects of other risk factors of noncommunicable diseases (tobacco use, unhealthy diet, physical inactivity, and harmful use of alcohol). In sum, aging-related health decline is the major cause of mortality and morbidity worldwide and should be addressed according to the severity of the problem. Because of these severe and negative effects, aging is already regarded as one of the greatest economic and societal challenges that most countries - especially in the industrialized world - will face in the coming decades.

Several reports from national governments, international organizations, such as the United Nations (UN) and WHO, as well as insurance companies, welfare organizations and non-governmental organizations (NGOs), have referred to the socio-economic and healthrelated challenges of aging societies [5-9]. These include:

$>$ Increases of health care costs and financial stress for social insurance systems due to the increasing probability of suffering from diseases associated with aging.

$>$ Dementia is often mentioned as especially problematic since it additionally requires intensive care-giving, imposing a heavy burden on working relatives or demanding significant additional costs for the provision of professional care.
A declining active workforce is already occurring, especially in industrialized countries. Although people might want to participate in the workforce for a longer time, they may not be able to, due to cognitive and physical decline.

$>$ An increasing number of pensioners need to be financed, while a larger number of working people need to provide care services that must remain affordable.

$>$ There are considerable concerns over the quality of life and health status of the elderly due to age-related diseases, biological degeneration and increased risk of succumbing to severe chronic and life-threatening conditions.

$>$ An overall decline in the quality of life deprives many elderly from full social, cultural and intellectual engagement.

The challenge of the aging society has been widely recognized and numerous research and development programs around the globe have been initiated to tackle age-related diseases, such as the Campaign to Prevent Alzheimer's Disease and/or WHO Diabetes Prevention Program, as well as to develop care-related technologies such as care robots, fall detectors and technologies for Ambient Assisted Living or making housing and infrastructure more friendly and accessible to the elderly. Although such investments are laudable and understandable, given the urgency of the problem, they all represent only punctual and rather ad hoc solutions.

In particular, assistive technologies, infrastructural changes or improving nursing and palliative care facilities, do not solve the actual problem of biological degeneration associated with the aging process. Medical research and development efforts currently are focused mainly on single diseases, like Alzheimer's dementia, heart disease, osteoporosis, diabetes, cancer, etc. The underlying degenerative aging processes, determinative for the emergence of those diseases, are often underemphasized.

While the degenerating aging process, involving the accumulation of structural damage, impairment of metabolic balance and functioning, is a disabling and debilitating process that requires prevention and treatment, the achievement of healthy longevity, characterized by the maintenance of functional capacity and robustness, is its cure.

Some effects of degenerative aging processes can be modified by social and economic factors, as well as lifestyles (diet, exercise, etc.), but only to a modest extent. Hence, there is a need to promote research into the biology of aging and aging-related diseases as the way to improve the health of the elderly more substantially. 
New directions in research and development take a more holistic approach for tackling the degenerative processes and negative biological effects of human aging, addressing several major fundamental causes of aging and aging-related diseases at once and in an interrelated manner. For example, at the 2013 US National Institutes of Health (NIH) Geroscience Summit, the following priority research areas have been identified: Adaptation to Stress, Epigenetics, Inflammation, Macromolecular Damage, Metabolism, Proteostasis, and Stem Cells/Regeneration [10,11], but there are several other examples of similar approaches, prioritizing research of major sets of aging processes [12-17]. Instead of targeting single age-related diseases, the mechanisms of the aging process itself are being analyzed with the goal of finding ways for intervention and prevention.

Such approaches are very promising, for the following reasons:

> They are already supported by scientific proofs of concept, involving the evidential increase in healthy lifespan in animal models and the emerging technological capabilities to intervene into fundamental aging processes [12,18-23].

$>$ They can provide solutions to a number of noncommunicable, age-related diseases, insofar as such diseases are strongly determined by degenerative aging processes (such as chronic inflammation, crosslinkage of macromolecules, somatic mutations, loss of stem cell populations, and others) [24-28]. Moreover, they are likely to decrease susceptibility of the elderly also to communicable diseases due to improvements in immunity [29].

$>$ The innovative, applied results of such research and development will lead to sustainable solutions for a large array of age-related medical and social challenges that may be globally applicable.

$>$ Such research and development should be supported on ethical grounds, to provide equal health care chances for the elderly as for the young.

Therefore it is the societal duty, especially of the professionals in biology, medicine, health care, economy and socio-political organizations to strongly recommend greater investments in research and development dealing with the understanding of mechanisms associated with the human biological aging process and translating these insights into safe, affordable and universally available applied technologies and treatments.

Thus there may be great benefits for society, the economy and our overall quality of life to prioritize and accelerate the research, development and innovation in the field, including the following:
Regenerative medicine - including the development of stem cell technologies and their products, in vivo and ex vivo regeneration of organs and tissues; controlled cell death, immuno-elimination of senescent or cancer cells.

$>$ Tissue engineering - culturing and assembling of a wide variety of replacement organs and tissues, vascular and non-vascular, using such methods as 3D tissue printing, biodegradable scaffolding, bioreactors or self-organization.

$>$ Regulation of whole body homeostasis, including regulators of the circadian rhythm, neurohumoral and molecular bioregulators.

$>$ Geroprotective substances - e.g. anti-glycemic, statins, anti-coagulant, anti-oxidant, hormonemodulating, mitochondria-modulating, antiinflammatory, probiotic and bioregulating medications.

> Detoxification at the cellular and molecular level e.g. chelators, enterosorbents, AGE-breakers, oxidoreductive depolymerization, immunoclearance, enzymatic clearance, etc.

$>$ Dietary supplementation - establishing dietary nutrient requirements for the elderly and during the entire life-course, e.g. using supplementation with vitamins, microelements, macroergics.

$>$ Gene therapy and gene modulation - e.g. safe genetic engineering, epigenetic and pharmacological stimulation of "Longevity Genes" (e.g. Sirtuins and FOXO), attenuation of "Decay Accelerating Factors" (DAF), manipulation of longevity promoting genetic pathways (e.g. mTOR pathway), stimulation of telomerase activity, RNA interference, genetic and epigenetic longevity sequencing and screening.

$>$ Nanomedicine - e.g. nanoparticles for drug delivery, emerging prototypes of artificial immunity and oxygen supply micro- and nano-devices, potentially devices for macromolecular tissue repair.

$>$ Artificial organ replacement and electrophysiological interfaces and stimulation - e.g. functional organ and limb prostheses, neuro-prostheses, advanced braincomputer interfaces, electrophysiological stimulation of impaired neurological and muscle functions.

$>$ Quantified self - comprehensive self-monitoring and personalized diagnosis of vitality and age-related conditions, calculating regimens for balanced and healthy nutrition and sufficient physical activity for the aged.

Data mining - large health data analytics, discovering effective candidate interventions, formal and quantitative as well as visually accessible and interactive models of aging processes and diseases and anti-aging interventions. 
Cryopreservation and chemopreservation - beneficial for organ or tissue transplantation and for a deeper insight into the physiology of aging.

Addressing aging-related debilitating processes through such biomedical means should become a new and powerful approach and medical standard for the prevention of non-communicable diseases, which affect most people at the later stages of life. The purpose of preventive medicine for the elderly, using advanced biomedical technologies, is to preserve health of an aging individual so as to prevent functional decline.

Governments should ensure the creation and implementation of the following policies to promote research into the biology of aging and aging-related diseases, for improving the health of the global elderly population:

\section{1) Funding:}

- Ensuring a significant increase of governmental and non-governmental funding for goal-directed (translational) research in preventing the degenerative aging processes, and the associated chronic non-communicable diseases and disabilities, and for extending healthy and productive life, during the entire life course.

\section{Specifically:}

- Dedicating a designated percentage of budget within relevant ministries, such as ministries of health and/or science, particularly in the divisions concerning research and treatment of noncommunicable chronic diseases.

- Dedicating a specific percentage of the profits of commercial pharmacological, biotechnology and medical technology companies to such research and development.

- Establishing relevant research grant programs on a competitive as well as goal-directed basis.

- Doubling of funding for such research every 5 years for the next 20 years.

\section{2) Incentives:}

- Developing and adopting legal and regulatory frameworks that give incentives for goal-directed research and development designed to specifically address the development, registration, administration and accessibility of drugs, medical technologies and other therapies that will ameliorate the aging processes and associated diseases and extend healthy life.
Specifically:

- Developing criteria for efficacy and safety of geroprotective therapies.

- Facilitating in silico and animal testing, and ethical safety-enhanced human testing of such therapies.

- Deploying and ensuring geroprotective therapies in the status of adjuvant and life-extending therapies.

- Providing a shortened approval pathway for therapies with high level of efficacy evidence in preclinical and early clinical trials, as well as in cases of advanced degenerative and seemingly futile conditions.

- Granting a special recognition, status and benefits to commercial and public entities engaged is such research and development.

\section{3) Institutions:}

- Establishing and expanding national and international coordination and consultation structures, programs and institutions to steer promotion of research, development and education on the biology of aging and associated diseases and the development of clinical guidelines to modulate the aging processes and associated aging-related diseases and to extend the healthy and productive lifespan for the population.

\section{Specifically:}

- Establishing Biogerontology specialty and courses in Biogerontology as a common part of university curriculum.

- Developing and disseminating geroprotective regiments, based on the best available evidence, as part of authoritative health recommendations.

- Establishing cooperative centers of excellence for fundamental, translational and applied studies, alongside centers for strategic analysis, forecast, education and policy development on aging and longevity research, at academic institutes and various governmental and supra-governmental agencies.

These measures are designed to reduce the burden of the aging process on the economy and to alleviate the suffering of the aged and the grief of their loved ones. On the positive side, if granted sufficient support, these measures can increase the healthy life expectancy for the elderly, extend their period of productivity and their interaction with society, and enhance their sense of enjoyment, purpose, equality and valuation of life.

\section{References}

[1] World Health Organization (2014). World Health Statistics - Large Gains in Life Expectancy. Accessed 
December 2014. Retrieved from: http://www.who.int/mediacentre/news/releases/2014/w orld-health-statistics-2014/en/.

[2] Human Mortality Database. Accessed December 2014. Retrieved from: http://www.mortality.org.

[3] United Nations, Department of Economic and Social Affairs, Population Division (2011). World Population Prospects: The 2010 Revision, CD-ROM Edition. Accessed December 2014. Retrieved from: http://esa.un.org/wpp/.

[4] World Health Organization (2013). Draft Twelfth General Programme of Work. Accessed December 2014. Retrieved

from: http://www.who.int/about/who_reform/programme_pri ority/en/.

[5] World Health Organization (2014). Ageing and Life Course. Accessed December 2014. Retrieved from: http://www.who.int/ageing/en/.

[6] United Nations, Department of Economic and Social Affairs, Population Division (2013). World Population Aging Report. Accessed December 2014. Retrieved from:

http://www.un.org/en/development/desa/population/.

[7] The International Longevity Center Global Alliance (2010). Global Aging Report. Threats to Longevity. A Call to Action. Accessed December 2014. Retrieved from: http://www.ilc-alliance.org/index.php/reports/.

[8] Lozano R, Naghavi M, Foreman K, Lim S, Shibuya K, Aboyans V, et al. (2012). Global and regional mortality from 235 causes of death for 20 age groups in 1990 and 2010: a systematic analysis for the Global Burden of Disease Study 2010. Lancet, 380:2095-2128.

[9] Lim SS, Vos T, Flaxman AD, Danaei G, Shibuya K, Adair-Rohani H, et al. (2012). A comparative risk assessment of burden of disease and injury attributable to 67 risk factors and risk factor clusters in 21 regions, 1990-2010: a systematic analysis for the Global Burden of Disease Study 2010, Lancet, 380:2224-2260.

[10] Kennedy BK, Berger SL, Brunet A, Campisi J, Cuervo AM, Epel ES, et al. (2014). Geroscience: linking aging to chronic disease. Cell, 59:709-713.

[11] Healthspan Campaign (2013). NIH Geroscience Interest Group (GSIG) Releases Recommendations from the October 2013 Advances in Geroscience Summit. Accessed December 2014. Retrieved from: http:/healthspancampaign.org/2014/02/27/nihgeroscience-interest-group-gsig-releasesrecommendations-october-2013-advances-gerosciencesummit/

[12] Fahy GM, West MD, Coles LS, Harris SB (Eds). The Future of Aging: Pathways to Human Life Extension. New York: Springer; 2010.

[13] Fontana L, Kennedy BK, Longo VD, Seals D, Melov S (2014). Medical research: treat ageing. Nature, 511:405407.

[14] López-Otín C, Blasco MA, Partridge L, Serrano M, Kroemer G (2013). The hallmarks of aging. Cell, 153:1194-1217.
[15] De Grey ADNJ, Rae M. Ending Aging. The Rejuvenation Breakthroughs That Could Reverse Human Aging in Our Lifetime. New York: St. Martin's Press; 2007.

[16] Goldman DP, Cutler D, Rowe JW, Michaud PC, Sullivan J, Peneva D, Olshansky SJ (2013). Substantial health and economic returns from delayed aging may warrant a new focus for medical research. Health Aff, 10:1698-1705.

[17] Rae MJ, Butler RN, Campisi J, de Grey ADNJ, Finch CE, Gough M, et al. (2010). The demographic and biomedical case for late-life interventions in aging. Sci Transl Med, 2:40cm21.

[18] Kanfi Y, Naiman S, Amir G, Peshti V, Zinman G, Nahum L, et al. (2012). The sirtuin SIRT6 regulates lifespan in male mice. Nature, 483:218-221.

[19] Atala A. Extending life using tissue and organ replacement (2008). Curr Aging Sci, 1:73-83.

[20] Baati T, Bourasset F, Gharbi N, Njim L, Abderrabba M, Kerkeni A, et al. (2012). The prolongation of the lifespan of rats by repeated oral administration of [60] fullerene. Biomaterials, 33:4936-4946.

[21] Kheir JN, Scharp LA, Borden MA, Swanson EJ, Loxley A, Reese JH, et al. (2012). Oxygen gas-filled microparticles provide intravenous oxygen delivery. Sci Transl Med, 4:140ra88.

[22] Shawn M. Douglas SM, Bachelet I, Church GM (2012). A logic-gated nanorobot for targeted transport of molecular payloads. Science, 335:831-834.

[23] Kennedy BK, Pennypacker JK (2014). Drugs that modulate aging: the promising yet difficult path ahead. Transl Res, 163:456-465.

[24] Chung HY, Cesari M, Anton S, Marzetti E, Giovannini S, Seo AY, et al. (2009). Molecular inflammation: Underpinnings of aging and age-related diseases. Ageing Res Rev, 8:18-30.

[25] Lunn JS, Sakowski SA, Hur J, Feldman EL (2011). Stem cell technology for neurodegenerative diseases. Ann Neurol, 70:353-361.

[26] Baylis D, Bartlett DB, Patel HP, Roberts HC (2014). Understanding how we age: insights into inflammaging. Longev Healthspan, 3:6.

[27] Dai DF, Chiao YA, Marcinek DJ, Szeto HH, Rabinovitch PS (2014). Mitochondrial oxidative stress in aging and healthspan. Longev Healthspan, 3:6.

[28] Zieman S, Kass D (2004). Advanced glycation end product cross-linking: Pathophysiologic role and therapeutic target in cardiovascular disease. Congest Heart Fail, 10:144-149.

[29] Bredenkamp N, Nowell CS, Blackburn CC (2014). Regeneration of the aged thymus by a single transcription factor. Development, 141:1627-1637. 